\title{
A comparative study of model fitting for estimating the overall efficiency of grid-connected photovoltaic inverters
}

\author{
J. Ramos-Teodoro ${ }^{1}$, F. Rodríguez ${ }^{1}$, M. Pérez ${ }^{2}$ and M. Berenguel ${ }^{1}$ \\ ${ }^{1}$ Department of Informatics \\ ${ }^{2}$ Department of Chemistry and Physics \\ CIESOL-ceiA3, University of Almería \\ Carretera Sacramento, s/n, 04120, Almería (Spain) \\ Phone/Fax number: +34 9500145 39, e-mail: jeronimo.rt@ual.es, frrodrig@ual.es, mperez@ual.es, beren@ual.es
}

\begin{abstract}
Determining the static overall efficiency of inverters is sometimes necessary for control o design purposes. As getting this information from the manufacturers' datasheets or certified laboratories might not be always viable, this paper addresses its estimation from direct measurements under actual operating conditions. Particularly, the Sandia Inverter Model has been taken as a paradigm of methodology and adapted to deal with the available data for an office building's photovoltaic system over the 20132017 period. Two unidimensional and two bidimensional models have been selected and compared to assess their goodness of fit on three inverters of the same kind of which the system consists. The best-case scenario corresponds to an exponential curve fitting, in which the R-square value increases over 0.95 , outperforming the other models.
\end{abstract}

Key words. PV performance, solar modules, Sandia Inverter Model, direct current, Atersa CICLO-3000.

\section{Introduction}

The worldwide amount of installed photovoltaic (PV) facilities has significantly increased over the last few years and its expected to play a key role in the future sustainable power system [1]. In this regard, rather a lot of attention is paid to their efficiency, which determines the profitability of this kind of projects and relies on a good performance of components such as PV modules or inverters [2]. Whereas for certain design applications, using a weighted single value for the efficiency is acceptable on other occasions, it is required to know a periodic estimate of an inverter's performance: consider, for example, the cases of energy management strategies for self-consumers where the decisions taken on an hourly base require to foreseen the energy yield by a PV field [3] or the design of facilities based on multilevel optimization [4]. In these cases, a static model for predicting the overall efficiency from the actual power and direct current (DC) voltage, which are the main operating conditions that affects an inverter's performance, improves the accuracy while keeping the computational burden low.

Although the overall efficiency is the result of the conversion efficiency and the inverter's maximum-power-point- tracking (MPPT) effectiveness, this latter one can up to certain point be neglected or just indirectly considered in the overall model according to Sandia National Laboratories [5]. They have already proposed the so-called Sandia Inverter Model, which indirectly provides a means to predict the overall efficiency [6]. Despite constituting a great starting to obtain a model from manufacturers' datasheets, some issues might arise if the information provided by them needs to be compared with the actual efficiencies, which is the case for the inverters below analyzed.

Considering the above, the contributions of this study are: (1) provision of a methodology for modelling the steadystate efficiency of grid-connected photovoltaic inverters, under actual operating conditions, from measured data; (2) a comparison of both unidimensional and bidimensional models, considering the Sandia Inverter Model for benchmarking; (3) parametrization of the models for Atersa CICLO-3000 inverters.

The rest of the paper is organized as follows: Section 2 is devoted to describing CIESOL's photovoltaic system and introducing the Sandia Inverter Model that will be used for benchmarking; Section 3 presents the modelling results for the three CIESOL's inverters; whereas the conclusions deduced from this study are recapped in Section 4.

\section{Materials and Methods}

\section{A. CIESOL's Photovoltaic System}

The Solar Energy Research Centre (CIESOL, http://www.ciesol.es) is located at the University of Almeria campus $\left(36.83^{\circ} \mathrm{N}, 2.41^{\circ} \mathrm{W}\right)$, in a building with the same name as the center: CIESOL building (Figure 1). This has a total surface area of $1071.92 \mathrm{~m}^{2}$, laid out on two floors, and incorporates several energy-saving passive and active strategies. A network of sensors allows recording and monitoring information to estimate CIESOL's energy consumption, climate conditions, and occupancy, among others. Readers are referred to Castilla et al.'s book, which contains a description of it, for further information [7]. 
Its PV field was designed and engineered by Atersa (https://www.atersa.com/en/). It counts with forty-two Atersa A-222P modules, facing south (azimuthal angle of $21^{\circ}$ east), with a surface area of $1.63 \mathrm{~m}^{2}$ and a slope angle of $22^{\circ}$, and distributed in three arrays of fourteen collectors each connected in series (Figure 1). Each array feeds an Atersa CICLO-3000 inverter where several electrical parameters are measured and sent to a database.

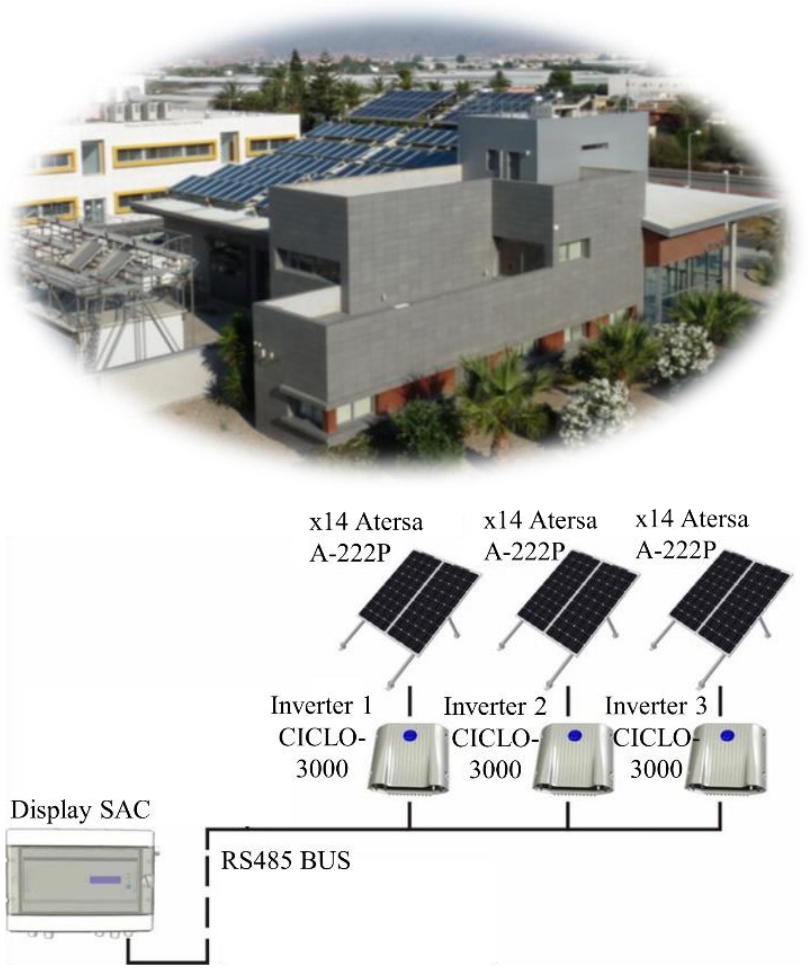

Fig. 1. CIESOL building and schematic of its PV system

The dataset employed in this study consist of minutely data from October 2013 to June 2017 for each inverter. The electrical measurements include DC current and voltage and alternate current and voltage from which the inverters' input and output power, as well as their conversion efficiencies, can be deduced. The main motivation for this analysis was to fit the models without using the manufacturer's efficiency curves, but from empirical data, so that a posterior comparison can be carried out.

\section{B. Data Cleansing}

From the 1368 days between the above-mentioned dates, only 104120 records are available for each inverter (a $52.85 \%$ of the potential total number). The rest of missing data correspond to periods of power outage, maintenance operations and other incidents that stopped the data acquisition system from recording.

For each inverter, cleansing was carried out in two stages: one regarding the manufacturer' datasheet, i.e. by selecting the records that satisfy Equations (1)-(4),

$$
\begin{gathered}
0 W \geq P_{d c} \leq 3000 W, \\
0 W \geq P_{a c} \leq 2500 W, \\
210 V \geq V_{d c} \leq 550, V \\
0 \geq \eta_{i n v} \leq 1,
\end{gathered}
$$

where $\boldsymbol{P}_{\boldsymbol{d} \boldsymbol{c}}$ and $\boldsymbol{V}_{\boldsymbol{d} \boldsymbol{c}}$ are the amount of DC input power and voltage, $\boldsymbol{P}_{\boldsymbol{a c}}$ is the amount of AC output power, and $\boldsymbol{\eta}_{\boldsymbol{i n} \boldsymbol{v}}$ is the overall efficiency calculated as the quotient of $\boldsymbol{P}_{\boldsymbol{a c}}$ and $\boldsymbol{P}_{\boldsymbol{d} \boldsymbol{c}}$. The other stage concerns the isolated samples that mostly correspond to abnormal data, which have been removed when they simultaneously met the criteria expressed in each row of Table I.

Table I. - Removing criteria for abnormal data

\begin{tabular}{|c|c|}
\hline Efficiency & DC Power \\
\hline $\boldsymbol{\eta}_{\text {inv }} \leq \mathbf{0 . 0 0 1}$ & $\boldsymbol{P}_{\boldsymbol{d c}}>\mathbf{0} \mathrm{W}$ \\
\hline $\boldsymbol{\eta}_{\text {inv }} \leq \mathbf{0 . 1}$ & $\boldsymbol{P}_{\boldsymbol{d c}}>\mathbf{2 0 0 \mathrm { W }}$ \\
\hline $\boldsymbol{\eta}_{\text {inv }} \leq \mathbf{0 . 7}$ & $\boldsymbol{P}_{\boldsymbol{d c}}>\mathbf{5 0 0 \mathrm { W }}$ \\
\hline $\boldsymbol{\eta}_{\boldsymbol{i n v}} \leq \mathbf{0 . 7 5}$ & $\boldsymbol{P}_{\boldsymbol{d c}}>\mathbf{1 0 0 0} \mathrm{W}$ \\
\hline $\boldsymbol{\eta}_{\text {inv }} \leq \mathbf{0 . 8}$ & $\boldsymbol{P}_{\boldsymbol{d c}}>\mathbf{1 5 0 0} \mathrm{W}$ \\
\hline
\end{tabular}

As a result of the above, Table II contains the total amount of records/samples considered for each inverter after cleansing. The percentage between rounded brackets refers to the total available amount of 104120 . Note that the first stage is much more noticeable in terms of samples because it indirectly filters the daylight periods.

Table II. - Samples considered for each inverter after cleansing

\begin{tabular}{|c|c|c|}
\hline Inverter & Stage 1 samples & Stage 2 samples \\
\hline 1 & $481944(46.29 \%)$ & $437138(41.99 \%)$ \\
\hline 2 & $486218(46.70 \%)$ & $444817(42.72 \%)$ \\
\hline 3 & $463443(44.51 \%)$ & $454791(43.68 \%)$ \\
\hline
\end{tabular}

\section{Sandia Inverter Model}

The Sandia Inverter Model provides a means to predict AC output power from DC input power, performing adjustments to consider different levels of DC input voltage, as given in Equations (5)-(8),

$$
\begin{gathered}
P_{a c}=\left[\frac{P_{a c 0}}{A-B}-C(A-B)\right]\left(P_{d c}-B\right)+C\left(P_{d c}-B\right)^{2},(5) \\
A=P_{d c 0}\left[1+C_{1}\left(V_{d c}-V_{d c 0}\right)\right], \\
B=P_{s 0}\left[1+C_{2}\left(V_{d c}-V_{d c 0}\right)\right], \\
C=C_{0}\left[1+C_{3}\left(V_{d c}-V_{d c 0}\right)\right],
\end{gathered}
$$

where $\boldsymbol{V}_{\boldsymbol{d c 0}}$ is the DC voltage level at which the AC power rating is achieved at reference operating conditions, $\boldsymbol{P}_{\boldsymbol{a c 0}}$ is the maximum $\mathrm{AC}$ power rating for inverter at reference conditions, $\boldsymbol{P}_{\boldsymbol{d c 0}}$ is the DC power level at which the AC power rating is achieved at reference operating conditions, $\boldsymbol{P}_{\boldsymbol{s} 0}$ is the DC power required to start the inversion process, $\boldsymbol{C}_{\mathbf{0}}$ is a parameter defining the curvature of the relationship between $\mathrm{AC}$ output power and DC input power, and $\boldsymbol{C}_{\mathbf{1}}, \boldsymbol{C}_{\mathbf{2}}$, and $\boldsymbol{C}_{\mathbf{3}}$ are empirical coefficients that respectively allow $\boldsymbol{P}_{\boldsymbol{d c 0}}, \boldsymbol{P}_{\boldsymbol{s} \mathbf{0}}$ and $\boldsymbol{C}_{\mathbf{0}}$ to vary linearly with DCvoltage input. As this model depends on both $\boldsymbol{P}_{\boldsymbol{d} \boldsymbol{c}}$ and $\boldsymbol{V}_{\boldsymbol{d} \boldsymbol{c}}$, it will henceforth be referred to as "Bidimensional Sandia Inverter Model", whereas the simplified case in which the effect of varying $\boldsymbol{V}_{\boldsymbol{d} \boldsymbol{c}}$ is neglected, by considering and $\boldsymbol{C}_{\mathbf{1}}$, $\boldsymbol{C}_{\mathbf{2}}$, and $\boldsymbol{C}_{3}$ equal to zero as in Ref. [5], will constitute the "Unidimensional Sandia Inverter Model". 


\section{Model fitting}

The algorithm to estimate the above parameters from inverter efficiency curves is summarized in Sandia's website [6] and further explained in one of their reports [5]. Manufacturers usually provide these curves at three different DC voltage levels, so that the mid-level value can be considered the reference level $\boldsymbol{V}_{\boldsymbol{d c 0}}$.

However, as no information on the CICLO-3000 inverters efficiency curves was used, a different approach needs to be considered by adapting Sandia's algorithm. First, the set of data for each inverter was divided into quartiles $(\mathrm{Q} 1, \mathrm{Q} 2$, Q3, Q4), considering $\boldsymbol{V}_{\boldsymbol{d} \boldsymbol{c}}$ as the dependent variable, to determine the three voltage levels. The minimum and maximum voltage levels are calculated as the mean of the data grouped in the first (Q1) and the fourth quartiles (Q4), whereas the reference level is given by the mean of the data belonging to the second and third quartiles (Q2 and Q3). Then, a second order polynomial can be fitted to the three sets of data (Q1, Q2-Q3, and Q4), and continue with Sandia's algorithm from step 3c [6]. Bear in mind that, according to the inverter's specifications, the value of $\boldsymbol{P}_{\boldsymbol{a c 0}}$ was fixed at $2500 \mathrm{~W}$ and the value of $\boldsymbol{P}_{\boldsymbol{s} \mathbf{0}}$ was corrected to $15 \mathrm{~W}$ because negative values were being obtained. This means that the effect of $\boldsymbol{V}_{\boldsymbol{d} \boldsymbol{c}}$ in Equation (7) was also neglected, by setting $\boldsymbol{C}_{\mathbf{2}}$ equal to zero.

MATLAB's function fit was employed to get the models presented in following section: polynomials of degree one and two were fitted at some steps of the Sandia's algorithm, another unidimensional model was obtained by directly fitting an exponential curve between $\boldsymbol{\eta}_{\boldsymbol{i n} \boldsymbol{v}}$ and $\boldsymbol{P}_{\boldsymbol{d} \boldsymbol{c}}$, whereas the last bidimensional model is the result of fitting a surface between $\boldsymbol{P}_{\boldsymbol{a} \boldsymbol{c}}, \boldsymbol{P}_{\boldsymbol{d} \boldsymbol{c}}$, and $\boldsymbol{V}_{\boldsymbol{d} \boldsymbol{c}}$, and then deriving the expression for $\boldsymbol{\eta}_{\text {inv }}$ (note that $\boldsymbol{\eta}_{\boldsymbol{i n v}}=\boldsymbol{P}_{\boldsymbol{a c}} / \boldsymbol{P}_{\boldsymbol{d c}}$ ).

\section{Results}

Figures 2-5 offer an at-a-glance comparison of the goodness of fit for each model and inverter. These include the parametrized expression that has been fitted from the data available, except for Figure 4, in which the surface corresponds to Equations (5)-(8), as described in Subsection 2. C. In this latter case, the parameters obtained for the Sandia Inverter Model are encompassed in Table III.

Table III. - Parameters of the Sandia Inverter Model

\begin{tabular}{|c|c|c|c|}
\hline Parameter & Inverter 1 & Inverter 2 & Inverter 3 \\
\hline $\boldsymbol{P}_{\boldsymbol{a c 0} \mathbf{0}}(\mathrm{W})$ & 2500 & 2500 & 2500 \\
\hline $\boldsymbol{P}_{\boldsymbol{d c 0} \mathbf{~}}(\mathrm{W})$ & 2596 & 2526 & 2637 \\
\hline $\boldsymbol{V}_{\boldsymbol{d c 0}}(\mathrm{V})$ & 380 & 384 & 377 \\
\hline $\boldsymbol{P}_{\boldsymbol{s} \mathbf{0}}(\mathrm{W})$ & 15 & 15 & 15 \\
\hline $\boldsymbol{C}_{\mathbf{0}}\left(\mathrm{W}^{-1}\right)$ & $5.87 \cdot 10^{-6}$ & $6.05 \cdot 10^{-5}$ & $3.07 \cdot 10^{-5}$ \\
\hline $\boldsymbol{C}_{\mathbf{1}}\left(\mathrm{V}^{-1}\right)$ & $1.39 \cdot 10^{-4}$ & $1.09 \cdot 10^{-4}$ & $1.30 \cdot 10^{-4}$ \\
\hline $\boldsymbol{C}_{\mathbf{2}}\left(\mathrm{V}^{-1}\right)$ & 0 & 0 & 0 \\
\hline $\boldsymbol{C}_{\mathbf{3}}\left(\mathrm{V}^{-1}\right)$ & 0.0466 & 0.0016 & -0.0018 \\
\hline
\end{tabular}

Although the mean error (ME), root mean square error (RMSE) and the R-square coefficient are also shown in Figures 2 -5 to give an idea of the goodness of fit, the distribution of the absolute error as a function of the DC input power has been included in Figure 6 as well, as in Ref. [5]. In general, Inverter 1 presents the worst results in terms of fitting (see in Figures 2-5) and the best modelling approach correspond to the exponential curves (see Figure 3). Also note that, in Figure 4, the correction to consider the linear effect of $\boldsymbol{V}_{\boldsymbol{d} \boldsymbol{c}}$ according to the modified Sandia's algorithm does not improve the results in Figure 2, possibly due to lack of data for wider DC voltage levels.

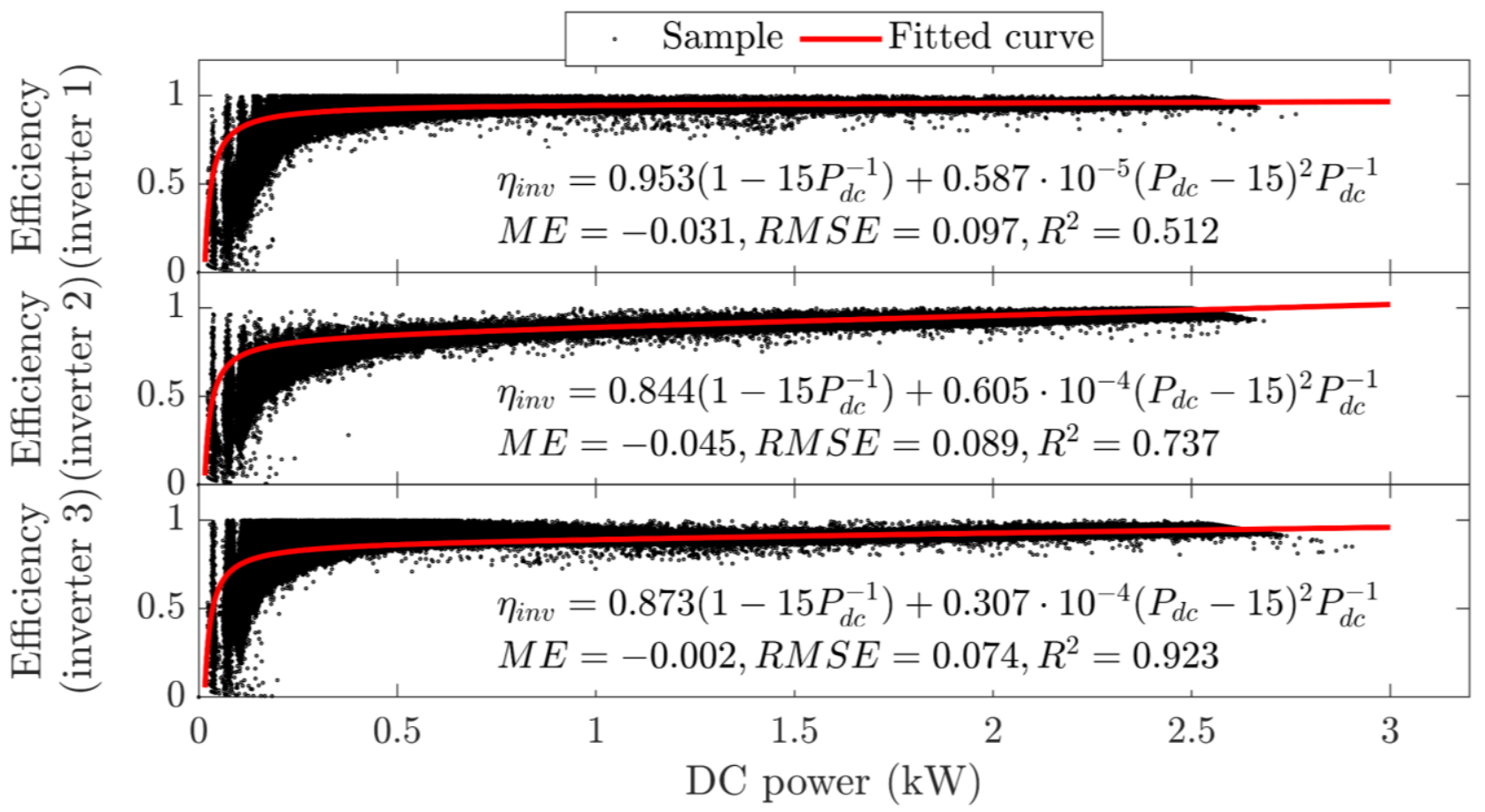

Fig. 2. Unidimensional Sandia Inverter Model (Model 1) 


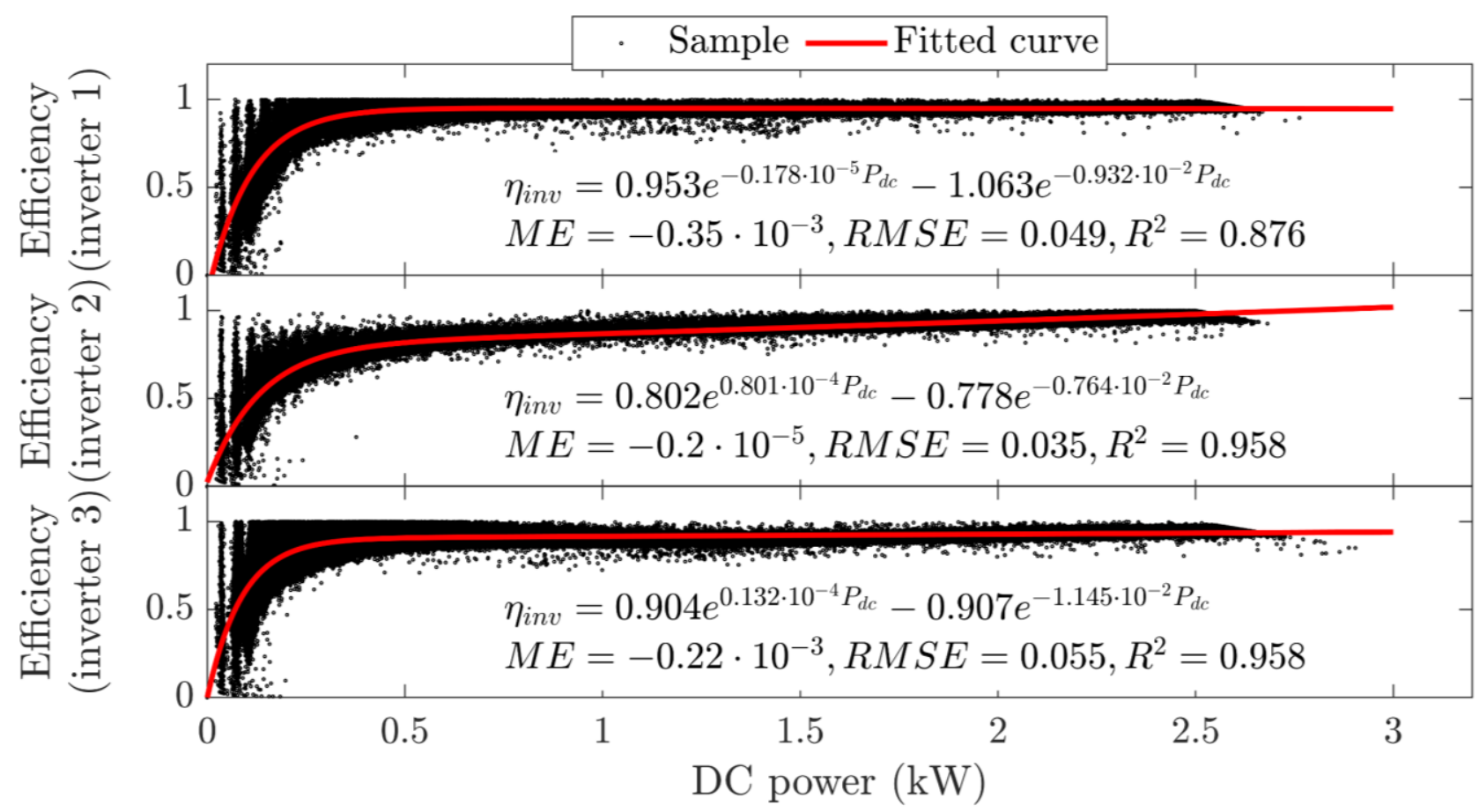

Fig. 3. Unidimensional exponential curve fitting (Model 2)

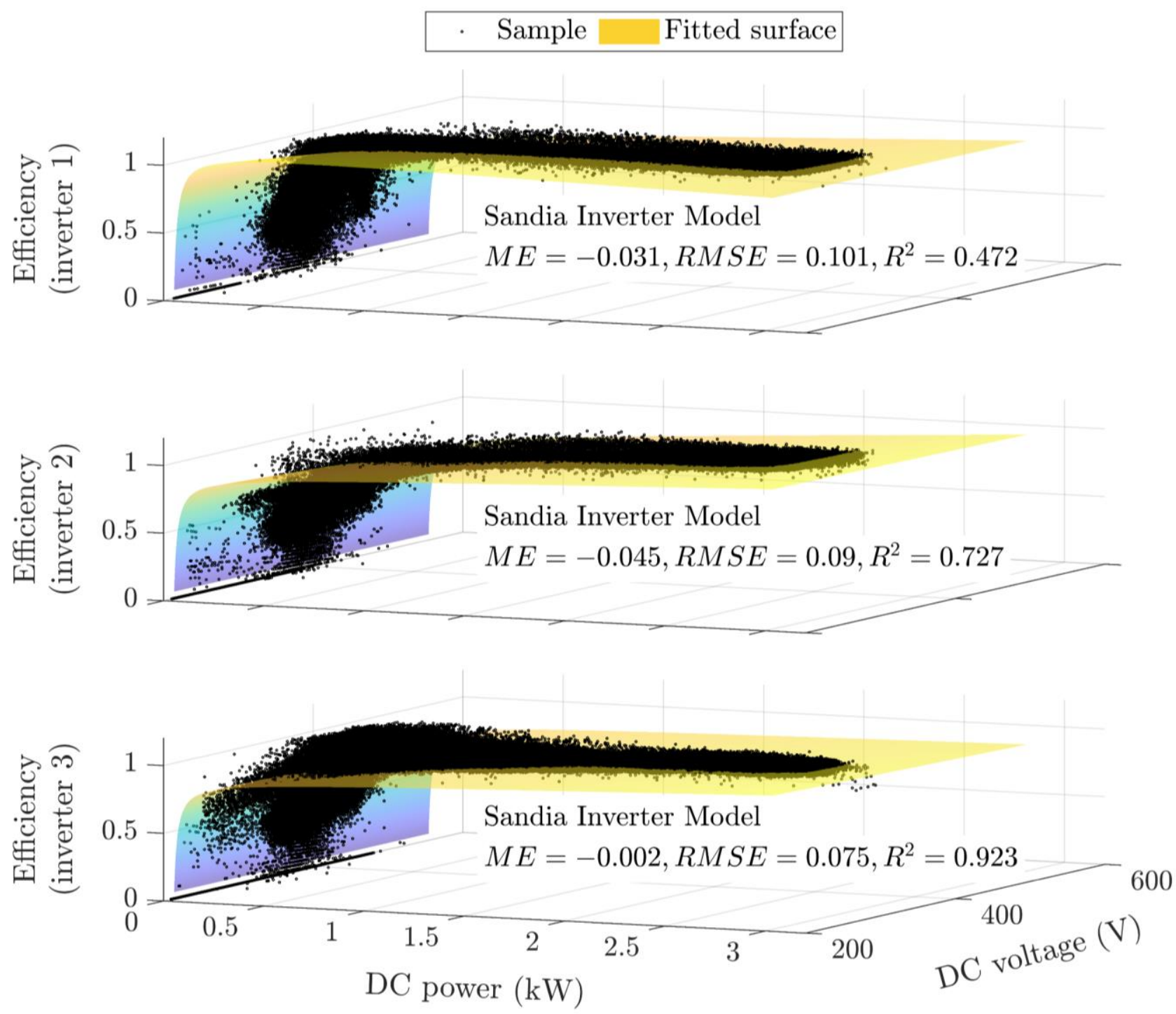

Fig. 4. Bidimensional Sandia Inverter Model (Model 3) 

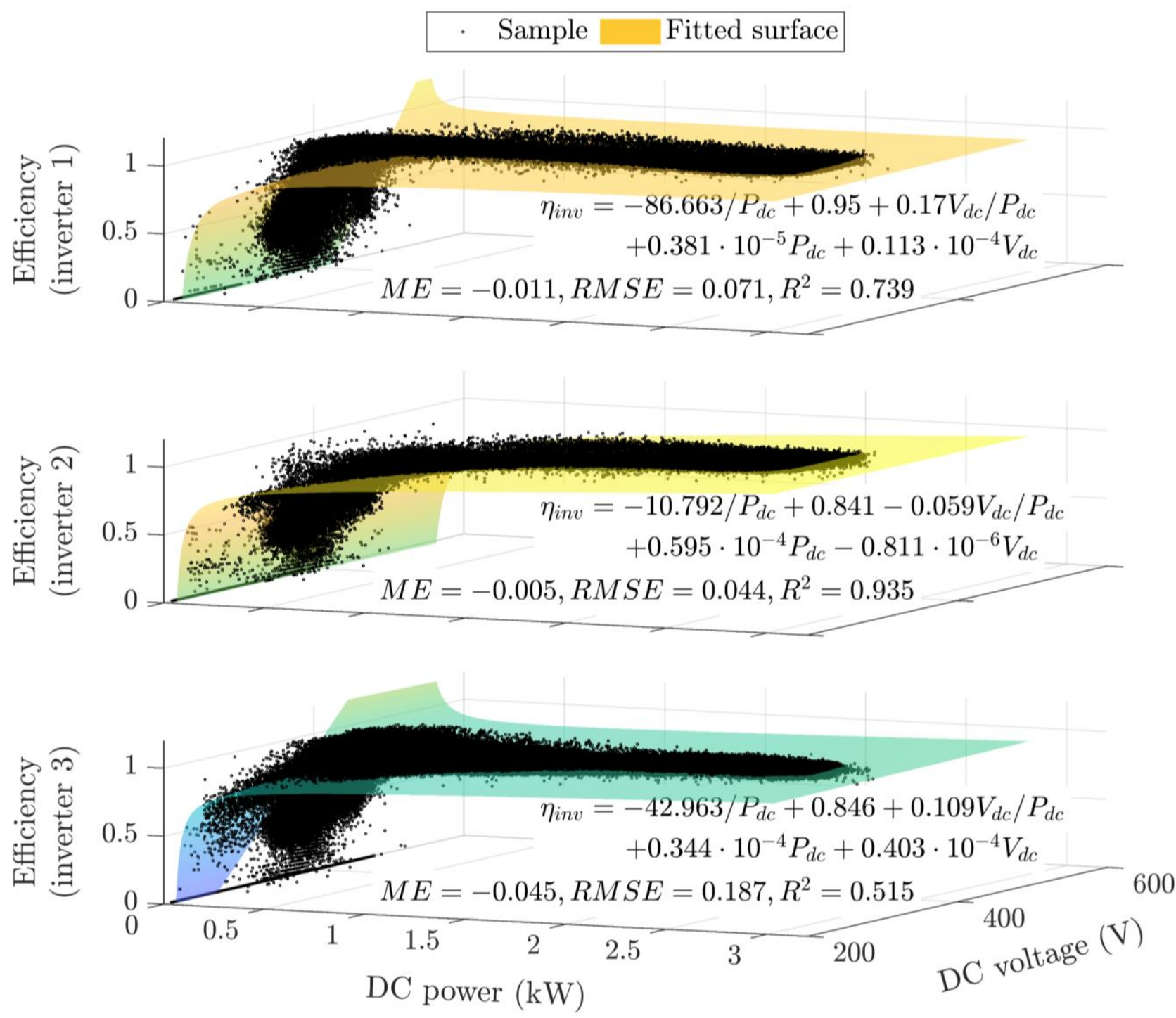

Fig. 5. Bidimensional surface fitting (Model 4)

On the contrary, the bidimensional model directly fitted (Figure 5) does seem to outperform the simplified Sandia Inverter Model in Figure 2. There is still some room for improvement in the case of Inverters 1 and 3, for which the fitted surfaces present asymptotic behaviors that yields bad estimates at low DC input power levels (especially for Inverter 3$)$.

In any case, all the models tend to slightly overestimate the efficiency as shown by the negative ME shown in Figures 2-5 and the error distribution in Figure 6, especially at lower DC input power levels when the uncertainty rises as well. The error is nevertheless well distributed around zero as in the case presented by Sandia National Laboratories [5], which contributes to prove the validity of this study.

\section{Conclusion}

Four different models were fitted and validated for CICLO3000 inverters in their actual operating conditions, by following the framework proposed by Sandia National Laboratories [5]. Although no efficiency curves from the manufacturer were used, the data available allowed grouping and generating them for three DC voltage levels.
The four models obtained will be refined in the future and compared against the efficiency curves obtained in a controlled environment for a wider range of voltages, although Model 2 has been in particular already used for a self-consumption facility [3]. On the one hand and in contrast to Sandia's approach, it provides the efficiency value from an exponential curve, so it might result fruitful to assess the fitting of exponential surfaces in this regard.

On the other hand, as the surfaces for Model 4 were actually estimated between $P_{a c}, P_{d c}$, and $V_{d c}$, it is not surprising the apparition of asymptotic behaviors when adapted to estimate $\eta_{\text {inv }}$. When parameter estimation is subject to solving a non-linear optimization problem, as is the case for hyperbolic or exponential surfaces, more complex fitting techniques will need to be used in the coming studies.

Finally, readers should be aware of one assumption that might require a more in-depth analysis: the one-minute sample period is supposed to ensure steady-state conditions as MPPT should take just some seconds to act [2], but if suddenly changing operating conditions occur, the data might require an additional filtering stage to exclude all the sample potentially corresponding to transients. 

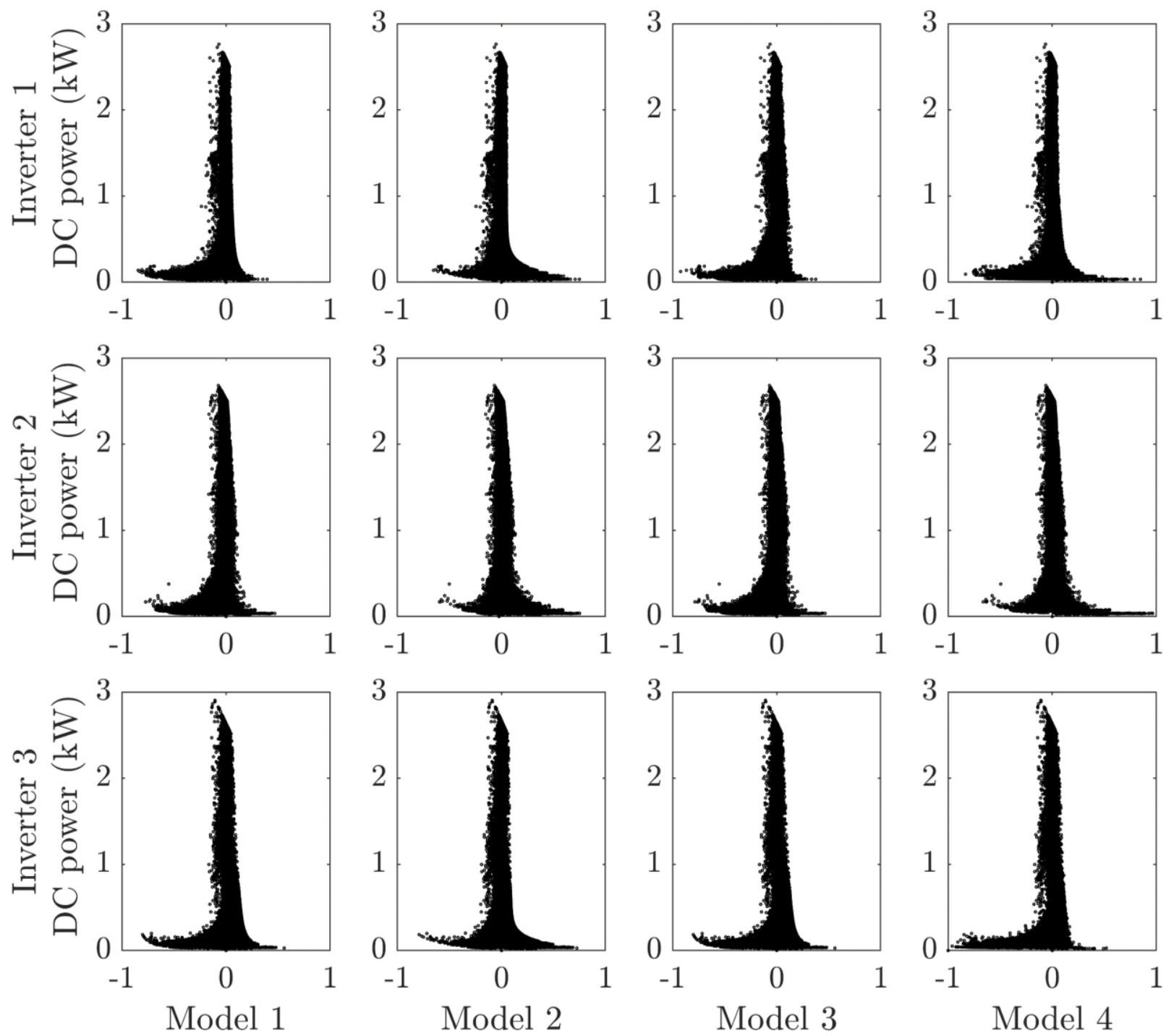

Absolute error in modeled efficency

Fig. 6. Error distribution as a function of DC power for each inverter and model analyzed

\section{Acknowledgement}

This work has been funded by the National R+D+i Plan Project DPI2017-85007-R of the Spanish Ministry of Science, Innovation and Universities and ERDF funds.

\section{References}

M. Gul, Y. Kotak, and T. Muneer, "Review on recent trend of solar photovoltaic technology," Energy Exploration \& Exploitation, vol. 34, no. 4, pp. 485-526, 2016.

[2] M. Valentini, A. Raducu, D. Sera, and R. Teodorescu, "PV inverter test setup for European efficiency, static and dynamic MPPT efficiency evaluation," in 11th International Conference on Optimization of Electrical and Electronic Equipment (OPTIM 2008), 2008, pp. 433-438.

[3] J. Ramos-Teodoro, F. Rodríguez, M. Berenguel, and J. L. Torres, "Heterogeneous resource management in energy hubs with self-consumption: Contributions and application example," Applied Energy, vol. 229, pp. 537-550, Nov. 2018.
R. Evins, "Multi-level optimization of building design, energy system sizing and operation," Energy, vol. 90, pp. 1775-1789, Oct. 2015.

[5] D. L. King, S. Gonzalez, G. M. Galbraith, and W. E. Boyson, "Performance Model for Grid-Connected Photovoltaic Inverters," Albuquerque, New Mexico, 2007.

[6] Sandia National Laboratories, "Sandia Inverter Model." [Online]. Available:

https://pvpmc.sandia.gov/modeling-steps/dc-to-acconversion/sandia-inverter-model/. [Accessed: 04Mar-2021].

[7] M. Castilla, J. D. Álvarez, F. Rodríguez, and M. Berenguel, "Comfort Control in Buildings," London: Springer, 2014, pp. 1-237. 